九州大学学術情報リポジトリ

Kyushu University Institutional Repository

\title{
Resistivity and offset error estimations for the small-loop electromagnetic method
}

\section{Sasaki, Yutaka}

Kyushu University, Department of Earth Resources Engineering

Son, Jeong-Sul

Korea Institute of Geoscience and Mineral Resources, Geotechnical Engineering

Kim, Changryol

Korea Institute of Geoscience and Mineral Resources, Geotechnical Engineering

Kim, Jung-Ho

Korea Institute of Geoscience and Mineral Resources, Geotechnical Engineering

http://hdl. handle. net/2324/25557

出版情報: Geophysics. 73 (3)，pp.F91-F95，2008-03-14. Society of Exploration Geophysicists バージョン：

権利関係: (C) 2008 Society of Exploration Geophysicists. 


\title{
Resistivity and offset error estimations for the small-loop electromagnetic method
}

\author{
Yutaka Sasaki ${ }^{1}$, Jeong-Sul Son ${ }^{2}$, Changryol $\mathrm{Kim}^{2}$, and Jung-Ho Kim ${ }^{2}$
}

\begin{abstract}
Handheld frequency-domain electromagnetic (EM) instruments are being used increasingly for shallow environmental and geotechnical surveys because of their portability and speed of use in field operations. However, in many cases, the quality of data is so poor that quantitative interpretation is not justified. This is because the small-loop EM method is required to detect very weak signals (the secondary magnetic fields) in the presence of the dominant primary field, so the data are inherently susceptible to calibration errors. Although these errors can be measured by raising the instrument high above the ground so that the effect of the conducting ground is negligible, it is impracticable to do so for every survey. We have developed an algorithm that simultaneously inverts small-loop EM data for a multidimensional resistivity distribution and offset errors. For this inversion method to work successfully the data must be collected at two heights. The forward modeling used in the inversion is based on a staggered-grid 3D finite-difference method; its solution has been checked against a 2.5D finite-element solution. Synthetic and real data examples demonstrate that the inversion recovers reliable resistivity models from multifrequency data that are contaminated severely by offset errors.
\end{abstract}

\section{INTRODUCTION}

The frequency-domain loop-loop electromagnetic (EM) method has been used for many years in mineral and groundwater exploration and for environmental investigations (Frischknecht et al., 1991; Fitterman and Labson, 2005). There are two types of loop-loop EM methods. The horizontal loop, or HLEM, method (also known as the Slingram technique) uses separate transmitter and receiver loops connected by a reference cable. The loop separation is varied to in- crease the depth of investigation. In the second type, the loops are rigidly fixed on a single board, and loop separation is very small compared with that in the HLEM method.

Recently, a new generation of this latter type of EM instrument has gained popularity for shallow engineering and environmental surveys (Won, 2003). Its advantages are: (1) portability and speed of use in field operation, (2) simultaneous acquisition of multiple frequency data, and (3) good lateral resolution.

Small-loop EM data commonly are interpreted qualitatively based on visual inspection of the secondary magnetic field. However, for many applications, information on both lateral and vertical variations in conductivity is required. Huang and Won (2003) argued that depth sounding is possible using their EM system to collect multifrequency data and showed synthetic and field data examples of a layered-model (1D) inversion. However, the quality of small-loop EM data is often not good enough for quantitative interpretation.

The small-loop EM system requires an accurately calibrated receiver loop to measure very weak signals (the secondary magnetic fields) in the presence of the dominant primary field. The problem is that even if an EM system is calibrated very precisely at the factory, calibration errors caused mainly by slight displacements of the fixed loops and electronic drift are inevitable in the field. The recommended procedure for correcting the EM signal is to measure calibration errors as a function of frequency by raising the sensor high above the ground so that measurement is not affected by the conducting ground (Won, 2003). This is not practical, however, in common field situations.

Another procedure recommended particularly for a single-frequency EM instrument is to obtain the instrument response as a function of height and determine calibration factors by comparing it with the response computed for a ground-truth model determined from independent geophysical data (Butler et al., 2004; Reid et al., 2004).

Simultaneous estimation of both resistivity and calibration error is relatively new. Konishi (2002) proposed a method that simultaneously inverts airborne EM data for a 1D resistivity model and calibration errors. This method determines calibration factors based on

Manuscript received by the Editor 15 August 2007; revised manuscript received 6 November 2007; published online 14 March 2008

${ }^{1}$ Kyushu University, Department of Earth Resources Engineering, Fukuoka, Japan. E-mail: sasaki@ mine.kyushu-u.ac.jp.

${ }^{2}$ Korea Institute of Geoscience and Mineral Resources, Geotechnical Engineering, Daejeon, Korea. E-mail: jsson@kigam.re.kr; ryol1102@kigam.re.kr; jungho@kigam.re.kr.

(C) 2008 Society of Exploration Geophysicists. All rights reserved. 
the data at a selected site and proceeds with conventional 1D inversion. Brodie and Sambridge (2006) described an approach that simultaneously inverts entire airborne EM data using 1D forward modeling to estimate a laterally continuous conductivity model and calibration factors.

In this paper, we present a method for simultaneously inverting small-loop EM data acquired at multiple heights for a multidimensional resistivity distribution and offset errors. Here, offset error refers to the type of calibration error caused by the instrument response not being zero in free space (Frischknecht et al., 1991). The forward modeling used in the inversion is based on a finite-difference (FD) method in which the EM fields are solved on a 3D staggered grid (Sasaki and Meju, 2006). The effectiveness of the inversion method is demonstrated by using synthetic and field data examples.

\section{INVERSION METHOD}

The subsurface is assumed to be divided into a set of rectangular blocks. Let the logarithms of the resistivities of the blocks be represented by the vector $\mathbf{m}$, and let observed data be the vector $\mathbf{d}$. The data consist of the in-phase and quadrature components of the secondary magnetic field normalized by the (free-space) primary field and are assumed to have been collected at multiple heights above the ground. When considering offset errors, the inverse problem can be written as

$$
\mathbf{d}=\mathbf{f}(\mathbf{m})+\mathbf{G s},
$$

where $\mathbf{f}(\mathbf{m})$ is the forward-modeling function that generates the theoretical response for the model $\mathbf{m}$; $\mathbf{s}$ represents the offset errors contained in the data, which are different for the in-phase and quadrature components for each frequency; and $\mathbf{G}$ is a matrix that relates offset errors to the data.

Most of the elements of $\mathbf{G}$ are zeros, but each row has a 1 at the appropriate location. Note that the size of $\mathbf{s}$ (the number of offset values) is twice the number of frequencies. Formally, equation 1 is similar to the equation used in the inversion of magnetotelluric (MT) data to remove static shifts (deGroot-Hedlin, 1991). However, unlike static shifts in MT data, offset errors in EM data are independent of the subsurface structure. Thus, the inversion of EM data requires no assumptions about the statistical distribution of offset errors.

The solution to equation 1 is numerically unstable in the presence of noise. The inverse problem therefore is formulated as an optimization problem in which the solution is taken as a model that minimizes the objective function

$$
\phi=\|\mathbf{W}[\mathbf{d}-\mathbf{f}(\mathbf{m})-\mathbf{G s}]\|^{2}+\lambda^{2}\|\mathbf{C m}\|^{2},
$$

where $\mathbf{W}$ is a diagonal matrix assigning weights to each datum, $\mathbf{C}$ is a second-order finite-difference operator used to quantify the model roughness, and $\lambda$ is a Lagrange multiplier. The optimization problem is solved iteratively using a linearized approximation of the model

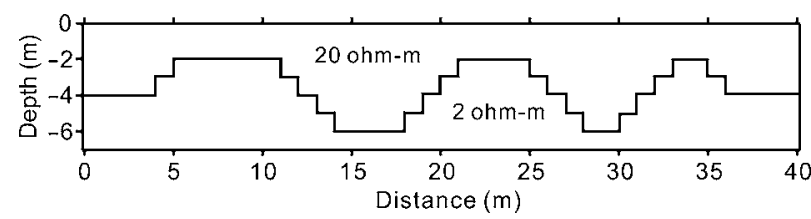

Figure $1.2 \mathrm{D}$ resistivity model used to verify accuracy of the FD solution and generate the synthetic data for inversion. The model is composed of two layers with an undulating boundary. function. Let $\Delta \mathbf{m}^{k}$ be the perturbation at the kth iteration to the current model $\mathbf{m}^{k-1}$. The objective function is approximated by

$$
\phi=\left\|\mathbf{W}\left[\Delta \mathbf{d}-\mathbf{J} \Delta \mathbf{m}^{k}-\mathbf{G s}\right]\right\|^{2}+\lambda^{2}\left\|\mathbf{C}\left(\mathbf{m}^{k-1}+\Delta \mathbf{m}^{k}\right)\right\|^{2},
$$

where $\mathbf{J}$ is the Jacobian matrix of sensitivities with respect to the model parameters, and

$$
\Delta \mathbf{d}=\mathbf{d}-\mathbf{f}\left(\mathbf{m}^{k-1}\right)
$$

is the vector of differences between the observed and predicted data. The minimization of $\phi$ is equivalent to obtaining the least-squares solution of the rectangular system

$$
\left[\begin{array}{cc}
\mathbf{W J} & \mathbf{W G} \\
\lambda \mathbf{C} & 0
\end{array}\right]\left\{\begin{array}{l}
\Delta \mathbf{m}^{k} \\
\mathbf{s}
\end{array}\right\}=\left\{\begin{array}{l}
\mathbf{W} \Delta \mathbf{d} \\
-\lambda \mathbf{C m}^{k-1}
\end{array}\right\} .
$$

If equation 5 is rewritten in compact form as

$$
\mathbf{A x}=\mathbf{y},
$$

the $n \times m$ matrix A can be decomposed using the modified GramSchmidt method or the Householder transformation into

$$
\mathbf{A}=\mathbf{Q R}
$$

where $\mathbf{Q}$ is an $n \times m$ orthogonal matrix (i.e., $\mathbf{Q}^{T} \mathbf{Q}=\mathbf{I}$ ) and $\mathbf{R}$ is an $m \times m$ upper triangular matrix (Bjorck, 1996). The solution is obtained by solving

$$
\mathbf{R x}=\mathbf{Q}^{T} \mathbf{y}
$$

which amounts to the backward substitution. The updated model is given by

$$
\mathbf{m}^{k}=\mathbf{m}^{k-1}+\Delta \mathbf{m}^{k} .
$$

The iteration is continued until the misfit measure is reduced to an acceptable level.

\section{VERIFICATION OF THE FORWARD SOLUTION}

Because multidimensional forward modeling for a small-loop EM survey is relatively new, the 3D FD solutions were checked against the 2.5D finite-element (FE) solutions of Kwon et al. (2002) for the model shown in Figure 1. The horizontal loops are assumed to be $2 \mathrm{~m}$ apart and on the ground surface.

The FD modeling employed a grid of $63 \times 29 \times 27$ (including 13 air layers) in the $x$-, $y$-, and $z$-directions, respectively. The smallest cell size used was $1 \times 2 \times 1 \mathrm{~m}$ in the central region, and the largest cell size was $128 \times 128 \times 128 \mathrm{~m}$ in the corners of the grid. The $2.5 \mathrm{D}$ FE modeling was carried out on a grid of $189 \times 66$ (including 18 air layers) in the $x$ - and $z$-directions, respectively, with the smallest cell size of $25 \times 25 \mathrm{~cm}$.

Figure 2 shows the comparisons of the 3D FD modeling result with the 2.5D FE solution. The in-phase and quadrature components of the secondary magnetic fields are plotted in parts per million (ppm) of the primary field at frequencies of 40,20,10, and $5 \mathrm{kHz}$. Although the discretization used for the 3D FD modeling was relatively coarse, the agreement is generally good. 


\section{NUMERICAL TESTS}

The inversion method was tested on the data generated for the model shown in Figure 1. The responses were computed at frequencies of 40,20,10, and $5 \mathrm{kHz}$ at 39 locations with an interval of $1 \mathrm{~m}$ and assuming loop separation of $2 \mathrm{~m}$ and heights of the loops above the ground of 1 and $2 \mathrm{~m}$. The FD modeling grid used was the same as that described above.

The computed responses were contaminated with Gaussian noise, with a standard deviation of $1 \%$ of the magnitude of the datum, except if this was less than 100 ppm, in which case 100 ppm was used. In addition, the data were shifted deliberately by a constant value at each frequency to simulate calibration errors. The shifted values are shown in the column with the heading "True" in Table 1.

In the inversions, the subsurface was divided into $280(40 \times 1 \times 7$ for the $x$-, $y$-, and $z$-dimensions, respectively) blocks of unknown resistivity. The Lagrange multiplier $\lambda$ was fixed at 0.2 throughout the inversion.

The result of inverting the data at a height of $1 \mathrm{~m}$ without accounting for offset errors is shown in Figure 3a. This resistivity image appears to be distorted considerably.

Next, the same data (at a single height) were inverted simultaneously for a 2D resistivity model and offset errors. The result (not shown) was far from the true model because of a trade-off between the model parameters and the offset parameters.

The simultaneous inversion also was applied to the data for heights of 1 and $2 \mathrm{~m}$. The inversions starting with homogeneous half-space models of resistivity 100 and $10 \mathrm{ohm}-\mathrm{m}$ produced the models shown in Figure $3 \mathrm{~b}$ and c, respectively. There is virtually no difference between the two inversion results, which implies that the inversion results are not dependent on the starting model. The offset errors are estimated almost correctly, as shown in Table 1 . The aver-
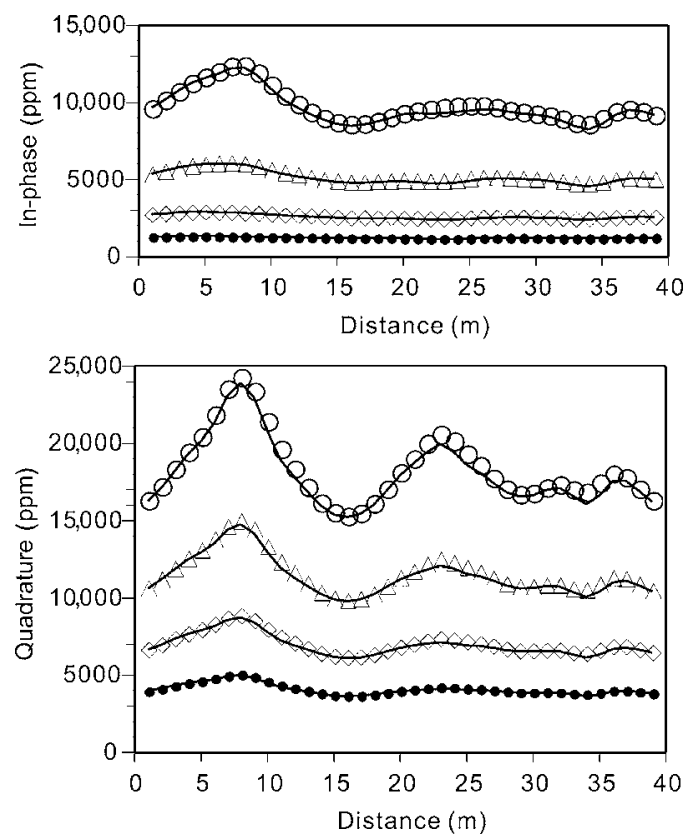

Figure 2. Comparison of the 3D FD results (symbols) with the $2.5 \mathrm{D}$ FE solution (solid lines) at four frequencies for the model shown in Figure 1. The in-phase (top) and quadrature (bottom) components of the secondary magnetic fields are normalized by the primary field. The frequencies used are $40 \mathrm{kHz}$ (open circles), $20 \mathrm{kHz}$ (triangles), $10 \mathrm{kHz}$ (diamonds), and $5 \mathrm{kHz}$ (solid circles). age data misfit reached $106 \mathrm{ppm}$ at the fifth iteration for both cases. This synthetic example clearly shows that offset errors can be estimated accurately by inversion if the profile data are acquired at two heights.

\section{FIELD EXAMPLE}

To examine the applicability of the method to real data, a smallloop EM survey using a GEM-2H EM sensor (Won et al., 1996) was carried out in the Yeonggwang coastal area of South Korea. Data were collected every $1 \mathrm{~m}$ at heights of 0 and $1 \mathrm{~m}$ above the ground along a 40-m survey line. Loop separation was $2.05 \mathrm{~m}$ along the line.

Table 1. Offset values assigned to the in-phase and quadrature components of the secondary magnetic field at four frequencies and those estimated by inversion. Cases 1 and 2 refer to inversion results obtained using homogeneous half-spaces with resistivities of 100 and $10 \mathrm{ohm}-\mathrm{m}$, respectively, as the starting model.

\begin{tabular}{lrrrrrrr} 
& \multicolumn{3}{c}{ In-phase $(\mathrm{ppm})$} & & \multicolumn{3}{c}{ Quadrature $(\mathrm{ppm})$} \\
\cline { 2 - 3 } $\begin{array}{l}\text { Frequency } \\
(\mathrm{kHz})\end{array}$ & True & Case 1 & Case 2 & & True & Case 1 & Case 2 \\
\hline 40 & -1500 & -1501 & -1501 & -3000 & -2790 & -2795 \\
20 & 1000 & 934 & 935 & -2500 & -2414 & -2417 \\
10 & 800 & 746 & 747 & -850 & -830 & -831 \\
5 & 500 & 446 & 447 & 1000 & 978 & 978 \\
\hline
\end{tabular}

a)

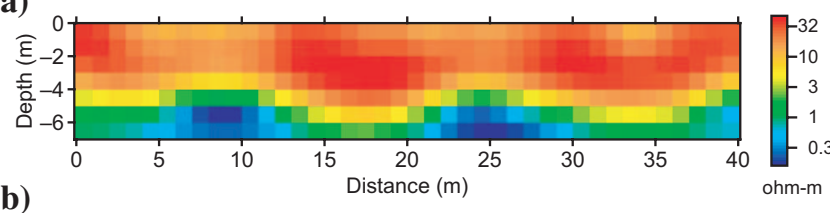

b)

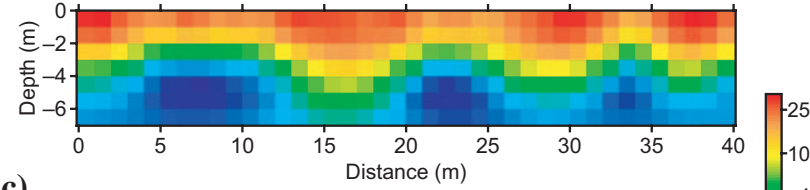

c)

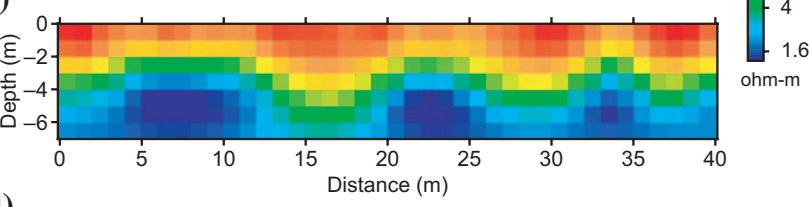

d)

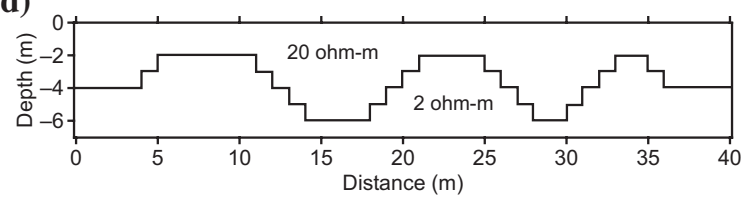

Figure 3. (a) Model obtained from inversion of the data at a height of $1 \mathrm{~m}$ without accounting for offset errors. (b) Model obtained from simultaneously inverting the data at heights of 1 and $2 \mathrm{~m}$ for resistivity and offset error, using a starting model of 100-ohm-m half-space. (c) Model obtained from simultaneously inverting the data at heights of 1 and $2 \mathrm{~m}$ for resistivity and offset error, using a starting model of 10-ohm-m half-space. (d) The true model. 
The profiles of EM data at frequencies of 40.11, 19.35, 9.33, and $4.53 \mathrm{kHz}$ at heights of 0 and $1 \mathrm{~m}$ are shown in Figures 4 and 5, respectively. Note that almost all the in-phase data have negative values, which cannot be handled in conventional inversions. A negative in-phase response can arise if the magnetic permeability of the earth materials is greater than that of free space (e.g., Beard and Nyquist, 1998). However, it is more likely to be caused by calibration errors because the value of the in-phase data becomes more negative as frequency increases, which cannot be explained by the effect of magnetic materials. Further, we have observed negative in-phase responses with the same instrument in many other fields.

The DC resistivity data also were recorded along the same line using a dipole-dipole array, with a dipole length of $1 \mathrm{~m}$ and a maximum $n$ spacing of 16 . The resistivity structures recovered from $2 \mathrm{D}$ inversions of EM and DC resistivity data are shown in Figure 6a and b, respectively. The starting model for EM inversion was a 100ohm-m half-space. A 20-ohm-m half-space also was tested as the starting model and produced a model (not shown) that was indistinguishable from the one shown in Figure 6a. The estimated calibration errors are shown in Table 2.

The agreement between the models in Figure $6 \mathrm{a}$ and $\mathrm{b}$ is not perfect, but considering that data errors manifest in very different ways and that the offset errors in EM data are quite large, the agreement is surprisingly good. The final fits between the observed data corrected for offset errors and the predicted data are shown in Figures 7 and 8. The average data misfits at the fifth iteration were 109 and 108 ppm for the starting models with 100- and 20-ohm-m half-spaces, respectively.

As a final experiment, the original field data were shifted deliberately by the same amount as in the synthetic example, and inversions were applied to the disturbed data using the same two starting models. The resulting models (not shown) were again virtually the same as the one shown in Figure 6a, and the recovered offset was nearly equal to the synthetic offset plus the previously recovered offset.
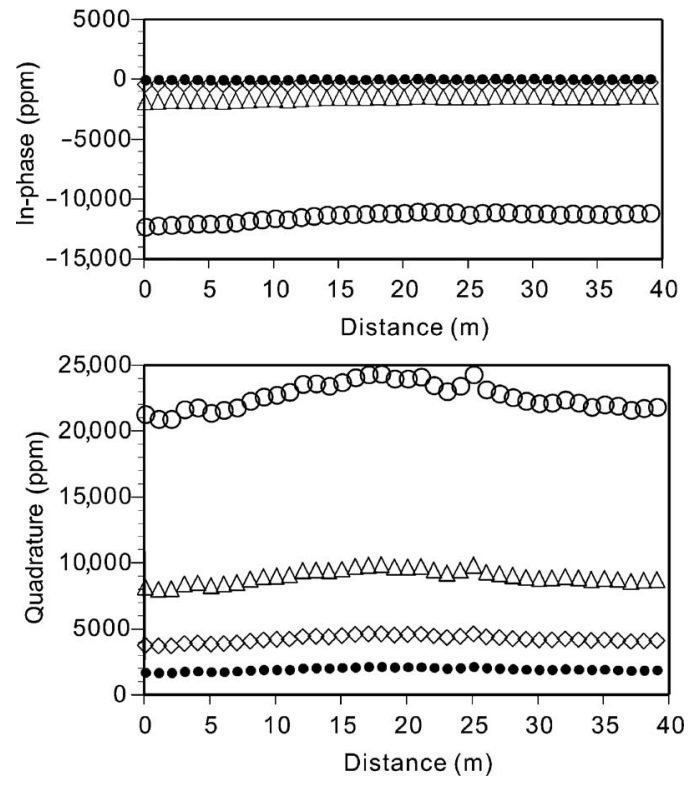

Figure 4. The in-phase (top) and quadrature (bottom) components of EM data collected at the surface. The frequencies used were $40.11 \mathrm{kHz}$ (open circles), $19.35 \mathrm{kHz}$ (triangles), $9.33 \mathrm{kHz}$ (diamonds), and $4.53 \mathrm{kHz}$ (solid circles).
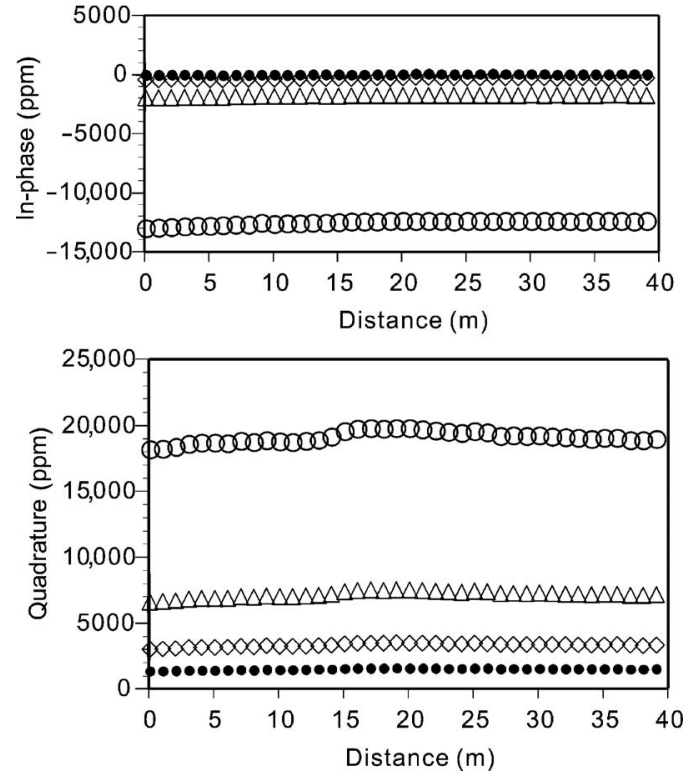

Figure 5. The in-phase (top) and quadrature (bottom) components of EM data collected at a height of $1 \mathrm{~m}$. The frequencies used were $40.11 \mathrm{kHz}$ (open circles), $19.35 \mathrm{kHz}$ (triangles), $9.33 \mathrm{kHz}$ (diamonds), and $4.53 \mathrm{kHz}$ (solid circles).

Table 2. Offset values estimated by inversion of EM data taken over the Yeonggwang coastal area. Cases 1 and 2 refer to inversion results obtained using homogeneous half-spaces with resistivities of 100 and $20 \mathrm{ohm}-\mathrm{m}$, respectively, as the starting model.

\begin{tabular}{cccccc}
\hline & \multicolumn{2}{c}{ In-phase $(\mathrm{ppm})$} & & \multicolumn{2}{c}{ Quadrature $(\mathrm{ppm})$} \\
\cline { 2 - 3 } \cline { 5 - 6 } $\begin{array}{c}\text { Frequency } \\
(\mathrm{kHz})\end{array}$ & Case 1 & Case 2 & & Case 1 & Case 2 \\
\hline 40.11 & -14263 & -14292 & & 12185 & 12226 \\
19.35 & -2627 & -2651 & & 3428 & 3438 \\
9.33 & -657 & -672 & & 1355 & 1353 \\
4.53 & -26 & -34 & & 519 & 514 \\
\hline
\end{tabular}

a)

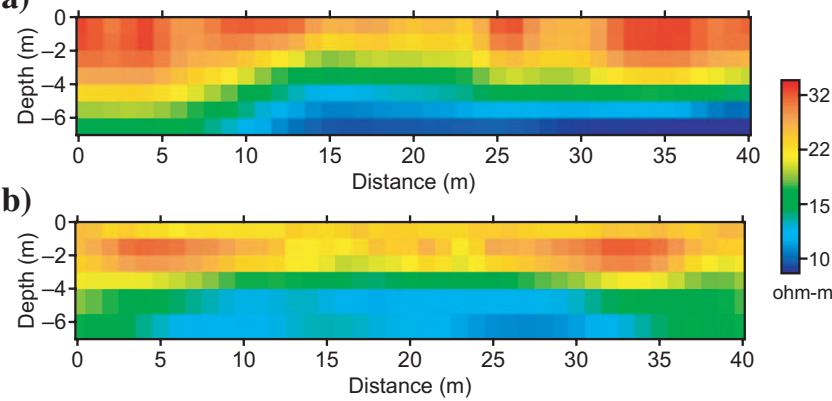

Figure 6. Models constructed from 2D inversions of EM (a) and dipole-dipole data (b). 
This experiment gives confidence in the reliability of this inversion approach for interpreting EM data seriously contaminated by offset errors.
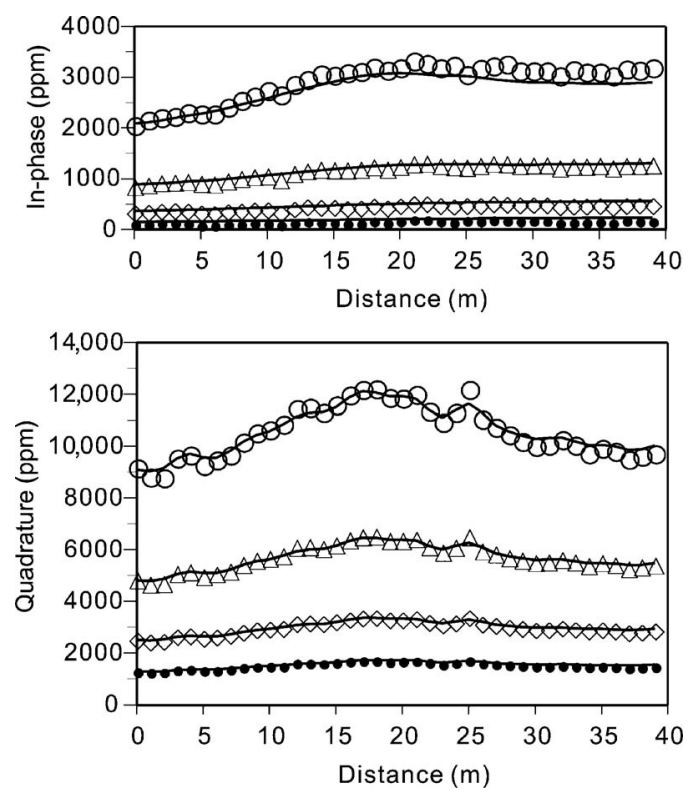

Figure 7. Comparisons between the observed data corrected for offset errors (symbols) and the predicted data (solid lines) at a height of $0 \mathrm{~m}$. The frequencies used were $40.11 \mathrm{kHz}$ (open circles), $19.35 \mathrm{kHz}$ (triangles), $9.33 \mathrm{kHz}$ (diamonds), and $4.53 \mathrm{~Hz}$ (solid circles).
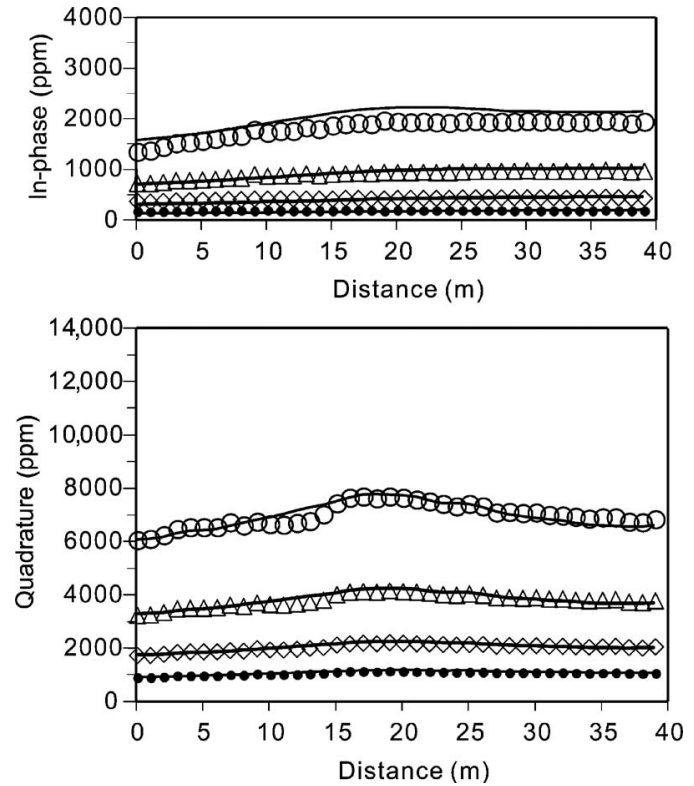

Figure 8. Comparisons between the observed data corrected for offset errors (symbols) and the predicted data (solid lines) at a height of $1 \mathrm{~m}$. The frequencies used were $40.11 \mathrm{kHz}$ (open circles), $19.35 \mathrm{kHz}$ (triangles), $9.33 \mathrm{kHz}$ (diamonds), and $4.53 \mathrm{kHz}$ (solid circles).

\section{CONCLUSIONS}

Offset errors can be a serious problem when quantitatively interpreting small-loop EM data. They can be measured and subtracted from the data if the instrument can be moved far enough from any conductor, but this is impracticable in most field situations.

We propose a practical approach that constructs reliable multidimensional models from raw data with offset errors. The prerequisite for this approach is to acquire the profile data at two heights. The proposed algorithm simultaneously inverts the data for resistivities and offset errors, imposing a constraint on the roughness of structure in the resistivity model.

The synthetic and field data examples discussed here demonstrate that even when data are contaminated severely by offset errors, the inversion recovers reliable resistivity models and is stable and independent of the starting model.

\section{ACKNOWLEDGMENTS}

This work was supported by the Basic Research Project of the Korea Institute of Geoscience and Mineral Resources, funded by the Ministry of Science and Technology of Korea. We thank James Reid and three anonymous reviewers for their helpful suggestions.

\section{REFERENCES}

Beard, L. P., and J. E. Nyquist, 1998, Simultaneous inversion of airborne electromagnetic data for resistivity and magnetic permeability: Geophysics, 63, 1556-1564.

Bjorck, A., 1996, Numerical methods for least squares problems: Society for Industrial and Applied Mathematics.

Brodie, R., and M. Sambridge, 2006, A holistic approach to inversion of frequency-domain airborne EM data: Geophysics, 71, no. 6, G301-G312.

Butler, K. E., J.-C. Nadeau, R. Parrott, and A. Daigle, 2004, Delineating recharge to a river valley aquifer by riverine seismic and EM methods: Journal of Environmental and Engineering Geophysics, 9, 95-109.

deGroot-Hedlin, C., 1991, Removal of static shift in two dimensions by regularized inversion: Geophysics, 56, 2102-2106.

Fitterman, D. V., and V. F. Labson, 2005, Electromagnetic induction methods for environmental problems, in D. K. Butler, ed., Near-surface geophysics: SEG, 301-355.

Frischknecht, F. C., V. F. Labson, B. R. Spies, and W. L. Anderson, 1991, Profiling methods using small sources, in M. N. Nabighian, ed., Electromagnetic methods in applied geophysics, v. 2: SEG 105-270.

Huang, H., and I. J. Won, 2003, Real-time resistivity sounding using a handheld broadband electromagnetic sensor: Geophysics, 68, 1224-1231.

Konishi, N., 2002, Development of a new drift correction technique for HEM leveling: Butsuri-Tansa (Geophysical Exploration), 55, 249-259 (in Japanese with English abstract).

Kwon, H.-S., Y.-H. Song, J.-S. Son, and J.-H. Suh, 2002, 2.5 dimensional EM modeling considering horizontal magnetic dipole source: Mulli-Tamsa (Geophysical Exploration), 5, 84-92 (in Korean with English abstract).

Reid, J. E., B. A. Kimber, and A. P. Worby, 2004, Calibration of a low induction number electromagnetic instrument for sea ice thickness measurements: Journal of Environmental and Engineering Geophysics, 9, 43-50.

Sasaki, Y., and M. A. Meju, 2006, A multidimensional horizontal-loop controlled-source electromagnetic inversion method and its use to characterize heterogeneity in a aquiferous fractured crystalline rocks: Geophysical Journal International, 166, 59-66.

Won, I. J., 2003, Small frequency-domain electromagnetic induction sensors: The Leading Edge, 22, 320-322.

Won, I. J., D. Keiswetter, G. Fields, and L. Sutton, 1996, GEM-2, A new multifrequency electromagnetic sensor: Journal of Environmental and Engineering Geophysics, 1, 129-137. 